\title{
A GENERIC TORELLI-TYPE THEOREM FOR SINGULAR ALGEBRAIC CURVES WITH AN INVOLUTION
}

\author{
MIROSLAV TSANOV JOTOV
}

(Communicated by Louis J. Ratliff, Jr.)

\begin{abstract}
We prove a generic Torelli-type theorem for a special class of singular algebraic curves with an involution. In order to obtain this result we introduce an appropriate mixed Hodge structure on the anti-invariant part of the first homology group, and study its properties.
\end{abstract}

Let $X$ be an irreducible projective algebraic curve with an involution $\sigma$. Suppose $X$ has only ordinary singularities and let $\Sigma$ be its singular locus. Let $\pi: N \rightarrow X$ be the normalization of $X$ and let $\tau: N \rightarrow N$ be the involution induced by $\sigma$. We suppose that the following condition is satisfied:

The set of fixed points of $\sigma$ coincides with $\Sigma$ and the involution $\tau$ is without fixed points.

Following J. Carlson [2] one can introduce a polarized mixed Hodge structure (PMHS) on the anti-invariant part of $H_{1}(X, \mathbb{Z})$ with respect to $\sigma$, denoted by $H_{1}^{-}(X, \mathbb{Z})$ :

$$
0 \rightarrow H_{1}^{-}(N, \mathbb{Z}) \rightarrow H_{1}^{-}(X, \mathbb{Z}) \rightarrow A \rightarrow 0,
$$

where $H_{1}^{-}(N, \mathbb{Z})$ is the anti-invariant part of $H_{1}(N, \mathbb{Z})$ with respect to $\tau$ and has a polarized Hodge structure (PHS) of weight $-1 ; A$ has PHS of weight 0 . It turns out that the latter is isomorphic to the lattice generated by a root-system of the type $D_{n}$ when $\#\left\{\pi^{-1}(\Sigma)\right\}>2$.

Using the generic Torelli theorem for the Prym map as proven by FriedmanSmith [4] and Kanev [5], the pair $(N, \tau)$ is uniquely determined by its Prym variety (equivalently by the PHS of $H_{1}^{-}(N, \mathbb{Z})$ ) if the following condition is satisfied.

$$
N / \tau \text { is a sufficiently general curve of genus } g \geq 7 \text {. }
$$

We prove the following result:

Theorem 7. Let $X$ be the curve which satisfies (*). Suppose that $N / \tau$ is a general curve of genus $g \geq 15$. Then $N, \tau$, and the set $\pi^{-1}(\Sigma)$ are uniquely determined by the PMHS of $H_{1}^{-}(X, \mathbb{Z})$.

Received by the editors October 1, 1989 and, in revised form, March 29, 1990.

1980 Mathematics Subject Classification (1985 Revision). Primary 14C30; Secondary 14H99.

Key words and phrases. Mixed Hodge structure, Prym variety, 1-motive map, root system. 
As a consequence we obtain

Theorem 8. If $X$ satisfies the conditions of Theorem 7 and has only one singular point then $X$ and $\sigma$ are uniquely determined by the PMHS of $H_{1}^{-}(X, \mathbb{Z})$.

\section{Preliminaries}

Let $X$ be an irreducible projective curve with ordinary singularities, and $n: N \rightarrow X$ be its normalization for which

(i) there exist involutions $\tau: N \rightarrow N$ and $\sigma: X \rightarrow X$, such that $\tau$ has no fixed points and the fixed points of $\sigma$ are the singularities of $X$;

(ii) $\pi \circ \tau=\sigma \circ \tau$.

Such curves can be constructed as follows:

(i) we fix $(N, \tau)$ to be a smooth irreducible projective algebraic curve with an involution without fixed points;

(ii) we choose a finite subset $\Omega \subset N$ such that $\Omega=\bigcup_{i=1}^{k} \Omega_{i}$, and for each $i, \tau\left(\Omega_{i}\right)=\Omega_{i}$ and $\Omega_{i} \cap \Omega_{j}=\varnothing$ if $i \neq j$;

(iii) we define $X=N / \rho$, provided with the factor-topology and the induced involution $\sigma$, where by definition for each $a, b \in N$

$$
\{a \rho b\} \text { iff }\left\{\text { either } a=b \text { or } a, b \in \Omega_{i} \text { for some } i\right\} \text {. }
$$

The condition that $X$ has only ordinary singularities uniquely determines $X$ as an algebraic curve.

Obviously $M=N / \tau$ is a smooth projective algebraic curve and the induced morphism $\psi: M \rightarrow Y=X / \sigma$ is a normalization. Furthermore $Y$ has at worst ordinary singularities.

Let $\phi: N \rightarrow M$ and $\lambda: X \rightarrow Y$ be the corresponding factor-morphisms.

\section{DEFINITION AND PROPERTIES OF THE MAIN EXACT SEQUENCE}

J. Carlson [2] constructed the following exact sequence for $\pi: N \rightarrow X$.

$$
0 \rightarrow H_{1}(N, \mathbb{Z}) \stackrel{\pi_{*}^{*}}{\rightarrow} H_{1}(X, \mathbb{Z}) \stackrel{\partial}{\rightarrow} \zeta_{x} \rightarrow 0,
$$

where $\partial$ is a boundary operator, $\zeta_{x} \subset \operatorname{Div}^{0} N$ is a finitely-generated group, which by means of the natural polarization on $\operatorname{Div} N$ (for which the points of $N$ form an orthonormal base) has a representation as an orthogonal sum: $\zeta_{x}=\bigoplus_{i=1}^{k} \zeta\left(y_{i}\right)$, where $y_{i}=\pi\left(\Omega_{i}\right)$. Furthermore $\zeta\left(y_{i}\right)$ is spanned by a rootsystem of type $A_{r(i)}$, where $r(i)=\#\left\{\Omega_{i}\right\}-1$. The morphisms $\sigma$ and $\tau$ act on (1). Since $\pi \circ \tau=\sigma \circ \pi$ for the anti-invariant part of the corresponding members of (1) we have:

$$
H_{1}^{-}(N, \mathbb{Z}) \stackrel{\pi_{*}}{\rightarrow} H_{1}^{-}(X, \mathbb{Z}) \stackrel{\partial}{\rightarrow} \zeta_{x} .
$$

Since $\tau\left(\Omega_{i}\right)=\Omega_{i}$ and $\tau$ has no fixed points we have $\zeta_{x}^{-}=\bigoplus_{i=1}^{k} \zeta^{-}\left(y_{i}\right)=$ $\bigoplus_{i=1}^{s} \mathbb{Z}\left(x_{i}-\tau\left(x_{i}\right)\right)$, which is an orthogonal sum. Here $2 s=\#\{\Omega\}$ and $\Omega=$ $\left\{x_{i}, \ldots, x_{s}, \tau\left(x_{i}\right), \ldots, \tau\left(x_{s}\right)\right\}$. Hence the nonzero elements of $\zeta_{x}^{-}$with min- 
imal length are

$$
\left\{ \pm\left(x_{i}-\tau\left(x_{i}\right) \mid x_{i} \in \Omega, \quad i=1,2, \ldots, s\right\} .\right.
$$

Let $A=\partial\left(H_{1}^{-}(X, \mathbb{Z})\right)$.

Lemma 1. (i) If $\#(\Omega)=2$, then $A \cong \mathbb{Z}, a$ and $(a, a)=8$;

(ii) If $\#\{\Omega\} \geq 4$ and $R=\left\{a \in \zeta_{x} \mid(a, a)=4\right\}$, then $R$ is a root-system of type $D_{s}(2 s=\#\{\Omega\})$ and $R$ generates $A$.

Proof. (a) We claim that there is no element $b \in \zeta_{x}^{-}$for which $(b, b)=2$ and $b \in A$. Indeed if there exists such an element then $b=x-\tau(x) \in A$ with $x \in \Omega$, hence there exists $c \in C_{1}(N)$ for which $\partial(c)=x-\tau(x)$ and $\pi_{*}(c) \in H_{1}^{-}(X, \mathbb{Z})$. Furthermore $c+\tau(c)$ is a cycle in $H_{1}(N, \mathbb{Z})$, and $\pi_{*}(c) \in$ $H_{1}^{-}(X, \mathbb{Z})$ implies that $\pi_{*}(c+\tau(c))=0$ in $H_{1}(X, \mathbb{Z})$. Since $H_{1}(N, \mathbb{Z}) \rightarrow$ $H_{1}(X, \mathbb{Z})$ is injective, it follows that $c+\tau(c)$ is homologous to 0 in $H_{1}(N, \mathbb{Z})$.

Now consider $\phi: N \rightarrow M$. Since $M=N / \tau$, it follows that $\phi_{*}(c+\tau(c))=$ $2 \phi_{*}(c)$ is homologous to 0 in $H_{1}(M, \mathbb{Z})$. Since $H_{1}(M, \mathbb{Z})$ is torsionfree, $\phi_{*}(c)$ is also homologous to 0 . Thus, as a loop in $\pi_{1}(M), \phi_{*}(c)$ is in the commutator subgroup. Since $\pi_{1}(N) \subset \pi_{1}(M)$ is a normal subgroup of index $2, \pi_{1}(N)$ contains the commutator subgroup. Thus $\phi_{*}(c)$ lies in the image of $\pi_{1}(N)$, which means that $\phi_{*}(c)$ lifts to a closed loop in $N$. This contradicts $\partial(c)=x-\tau(x)$.

(b) We claim that if $x, y \in \Omega$, then $a=x-\tau(x) \pm(y-\tau(y))$ is an element of $A$. It is sufficient to prove the statement for $a=x-\tau(x)+y-\tau(y)$ since $x-\tau(x)-(y-\tau(y))=x-\tau(x)+\tau(y)-\tau(\tau(y))$. Let $c \in C_{1}(N)$ with $\partial(c)=x-\tau(y)$. Then $\partial(c-\tau(c))=x-\tau(x)+y-\tau(y)$.

We complete the proof of Lemma 1 as follows:

Case (i). By (a) and (b) $A$ is generated by $a=2(x-y)$, where $\{x, y\}=\Omega$. Case (ii). Put $e_{i}=x_{i}-\tau\left(x_{i}\right)$, where $\Omega=\left\{x_{1}, \ldots, x_{s}, \tau\left(x_{1}\right), \ldots, \tau\left(x_{s}\right)\right\}$. Then $R=\left\{e_{i} \pm e_{j} \mid i \neq j\right\}$, so $R$ is a root-system of type $D_{s}$. Since $R=\left\{e_{i} \pm e_{j}\right\}$, it follows that $R$ spans a sublattice $R^{\prime}$ of index 2 in $\bigoplus_{i=1}^{s} \mathbb{Z} e_{i}$. Then (b) implies $R^{\prime} \subset A$, so that $R^{\prime}=A$ or $A=\bigoplus_{i=1}^{s} \mathbb{Z} e_{i}$. By (a), the latter cannot happen, which proves that $R$ generates $A$. Q.E.D.

Lemma 2. The sequence

$$
0 \rightarrow H_{1}^{-}(N, \mathbb{Z}) \stackrel{\pi_{*}}{\rightarrow} H_{1}^{-}(X, \mathbb{Z}) \stackrel{\partial}{\rightarrow} A \rightarrow 0
$$

is exact.

Proof. The exactness at the first member of (2) is derived from the exactness of the first member of (1); the exactness in the third member of (2) is derived from the definition of $A$. It remains to prove that $\operatorname{Ker} \partial=\operatorname{Im} \pi_{*}$. Obviously $\operatorname{Ker} \partial \supset \operatorname{Im} \pi_{*}$. Let $a \in H_{1}^{-}(X, \mathbb{Z})$ and $\partial(a)=0$, hence there exists $b \in$ $H_{1}(N, \mathbb{Z})$ such that $\pi_{*}(b)=a$, since $(1)$ is exact. We have

$$
0=a \sigma_{*}(a)=\pi_{*}(b)+\sigma_{*} \pi_{*}(b)=\pi_{*}(b+\tau(b)),
$$


hence $b+\tau(b)=0$, since $\pi_{*}$ is an injection. Hence $b \in H_{1}^{-}(N, \mathbb{Z})$ and $\pi_{*}(b)=a$, i.e., $\operatorname{Ker} \partial \subset \operatorname{Im} \pi_{*}$. Q.E.D.

\section{Construction of PMHS FOR $H_{1}^{-}(X, \mathbb{Z})$}

From the Poincare duality we get an exact sequence:

$$
0 \rightarrow \widehat{A} \stackrel{\widehat{\partial}}{\rightarrow} H_{-}^{1}(X, \mathbb{Z}) \stackrel{\pi^{*}}{\rightarrow} H_{-}^{1}(N, \mathbb{Z}) \rightarrow 0,
$$

where $\hat{A}=\operatorname{Hom}_{\mathbb{Z}}(A, \mathbb{Z})$.

Following J. Carlson [2] we define a mixed Hodge structure on $H_{-}^{1}(X, \mathbb{Z})$ :

(a) Weight filtration on $H_{-}^{1}(X, \mathbb{Z})$ :

$$
W_{-1}=0, \quad W_{0}=\operatorname{Im} \hat{\partial}, \quad W_{1}=H_{-}^{1}(X, \mathbb{Z}) ;
$$

define polarizations on $W_{0}$ and $W_{1} / W_{0}$ via the polarizations on $A$ and on $H_{1}^{-}(N, \mathbb{Z})$ introduced above.

(b) Hodge filtration on $H_{-}^{1}(X, \mathbf{C})=H_{-}^{1}(X, \mathbb{Z}) \otimes_{\mathbb{Z}} \mathbf{C}$ :

$$
\begin{aligned}
& F^{0}=H_{-}^{1}(X, \mathbf{C}) ; \\
& F^{1}=\left\{\omega \in H^{0}\left(X-\Sigma, \Omega^{1}(X-\Sigma)\right) \mid \int_{X-\Sigma} \omega \wedge \bar{\omega}<\infty, \sigma^{*} \omega+\omega=0\right\},
\end{aligned}
$$

here $\Sigma$ is the singular locus of $X$.

$F^{2}=0$.

Lemma 3. The map $\pi^{*}$ gives an isomorphism

$$
F^{1} \cong H_{-}^{0}\left(N, \Omega_{N}^{1}\right) .
$$

It follows from Lemma 3 that $F^{1} \cap W_{0}=0, W_{1} / W_{0} \otimes_{\mathbb{Z}} \mathbf{C} \cong F^{1} \oplus \overline{F^{1}}$ which means that we have MHS on $H_{-}^{1}(X, \mathbb{Z}), W_{0}$ has PHS of pure weight 0 , and $W_{1} / W_{0}$ has PHS of pure weight 1 (cf. [2]).

Proof. Let $\alpha \in F^{1}$. Then $\pi^{*}(\alpha) \in H^{0}\left(N-\Omega, \Omega^{1}(N-\Omega)\right)$. Since

$$
\int_{N-\Omega} \pi^{*} \alpha \wedge \overline{\pi^{*} \alpha}=\int_{X-\Omega} \alpha \wedge \bar{\alpha}<\infty,
$$

then $\pi^{*} \alpha \in H^{0}\left(N, \Omega_{N}^{1}\right)$. We have $\pi^{*} \alpha+\tau^{*} \circ \pi^{*} \alpha=\pi^{*}\left(\alpha+\sigma^{*} \alpha\right)=0$ on $N-\Omega$. The 1-form $\pi^{*} \alpha$ is holomorphic, so $\pi^{*} \alpha+\tau^{*} \circ \pi^{*} \alpha=0$ on $N$. Thus we have a linear map $\pi^{*}: F^{1} \rightarrow H_{-}^{0}\left(N, \Omega_{N}^{1}\right)$. The inverse of this map is obviously defined since $N$ and $X$ are birationally isomorphic. Q.E.D.

Proposition 4. The group $H_{1}^{-}(X, \mathbb{Z})$ has a polarized mixed Hodge structure for which:

(i) $W_{0} H_{1}^{-}(X)=H_{1}^{-}(X, \mathbb{Z}), W_{-1} H_{1}^{-}(X)=\operatorname{Im} \pi_{*}=H_{1}^{-}(N, \mathbb{Z}), W_{-2} H_{1}^{-}(X)$ $=0$; 
(ii) $F^{1} H_{1}^{-}(X)=0, F^{0} H_{1}^{-}(X)=\operatorname{ann}_{\mathbf{R}}\left(F^{1} H_{-}^{1}(X)\right) \cong \overline{F^{1} H_{-}^{1}(X)^{*}} \oplus\left(A \otimes_{\mathbb{Z}} \mathbf{C}\right)$, $F^{-1} H_{1}^{-}(X)=H_{1}^{-}(X, \mathbf{C})$, here $F^{1} H_{-}^{1}(X)^{*}=\operatorname{Hom}_{\mathbf{R}}\left(F^{1} H_{-}^{1}(X), \mathbf{R}\right)$ and the complex conjugation in $H_{-}^{1}(X, \mathbf{C})$ is induced by those in $\mathbf{C}$ through $H_{-}^{1}(X, \mathbf{C})$ $=H_{-}^{1}(X, \mathbb{Z}) \otimes_{\mathbb{Z}} \mathbf{C}$

(iii) $\mathrm{Gr}_{-1}^{w}=W_{-1} / W_{-2}$ has a pure weight -1 , the polarization of $H_{1}^{-}(N, \mathbb{Z})$ is transferred to $\mathrm{Gr}_{-1}^{w}$ through $\pi_{*}$.

$\mathrm{Gr}_{0}^{w}=W_{0} / W_{-1}$ has a pure weight 0 and the polarization of $A$ is transferred to $\mathrm{Gr}_{0}^{w}$ through $\partial$.

Proof. This is an immediate consequence of Lemma 3 and the definition of MHS on a dual group (cf. [2]). Q.E.D.

It follows that the Hodge structure on $\mathrm{Gr}_{-1}^{w}=W_{-1} H_{1}^{-}(X)$ is:

$$
\begin{aligned}
F^{-1} W_{-1} H_{1}^{-}(X) & =F^{-1} H_{1}^{-}(X) \cap\left[W_{-1} H_{1}^{-}(X) \otimes_{\mathbb{Z}} \mathbf{C}\right] \\
& =F^{1} H_{-}^{1}(X)^{*} \oplus \overline{F^{1} H_{-}^{1}(X)^{*}} \\
& =W_{-1} H_{1}^{-}(X) ; \\
F^{0} W_{-1} H_{1}^{-}(X) & =F^{0} H_{1}^{-}(X) \cap\left[W_{-1} H_{1}^{-}(X) \otimes_{\mathbb{Z}} \mathbf{C}\right]=\overline{F^{1} H_{-}^{1}(X)^{*}} ; \\
F^{1} W_{-1} H_{1}^{-}(X) & =0 .
\end{aligned}
$$

\section{GEOMETRIC DESCRIPTION OF THE 1-MOTIVE MAP}

By definition we have

$$
\begin{aligned}
& L^{0} H_{1}^{-}(X)=\left[\mathrm{Gr}_{0}^{w} H_{1}^{-}(X) \otimes_{\mathbb{Z}} \mathrm{C}\right] \cap\left[\mathrm{Gr}_{0}^{w} H_{1}^{-}(X)\right]_{\mathbb{Z}}, \\
& J^{0} W_{-1} H_{1}^{-}(X)=\left[W_{-1} H_{-}^{1}(X) \otimes_{\mathbb{Z}} \mathbf{C}\right] /\left[W_{-1} H_{1}^{-}(X)+F^{0} W_{-1} H_{1}^{-}(X)\right] .
\end{aligned}
$$

It is clear from Proposition 4 and from the sequence $(2)$ that $H_{1}^{-}(X, \mathbb{Z})$ is an extension of $\mathrm{Gr}_{0}^{w}$ by $\mathrm{Gr}_{-1}^{w}$. For extensions of this type J. Carlson [2] has constructed a map 1-motive:

$$
u: L^{0} H_{1}^{-}(X) \rightarrow J^{0} W_{-1} H_{1}^{-}(X),
$$

which depends only on the mixed Hodge structure of $H_{1}^{-}(X, \mathbb{Z})$ and is given as follows:

(i) Let $\left\{\omega_{i}\right\}$ be a basis of $\mathrm{Gr}_{-1}^{w}$ and $\left\{\omega^{i}\right\}$ be the dual basis in $\left(\mathrm{Gr}_{-1}^{w}\right)^{*}$;

(ii) Let $\left\{\Omega^{i}\right\} \subset H_{1}^{-}(X, \mathbb{Z})$, for which $\pi^{*}\left(\Omega^{i}\right)=\omega^{i}$ for each $i$;

(iii) If $\gamma \in \mathrm{Gr}_{0}^{w}$ and $\Gamma \in H_{1}^{-}(X, \mathbb{Z})$ is such that $\partial(\Gamma)=\gamma$, then $\gamma \stackrel{u}{\rightarrow}\left[\Sigma_{i}\right.$ $\left.\left\langle\Omega^{i}, \Gamma\right\rangle \omega_{i}\right]$, where $\langle$,$\rangle is the canonical pairing between H_{1}^{-}(X, \mathbb{Z})$ and $H_{-}^{1}(X, \mathbb{Z})$; and $[\alpha]$ is the class of $\alpha$ in $J^{0} W_{-1} H_{1}^{-}(X)$. 
Let us recall that for $(N, \tau)$ a Prym variety $P(N, \tau)=H_{-}^{1,0}(N)^{*} / H_{1}^{-}(N, \mathbb{Z})$ is defined and a map $P A: \operatorname{Div}^{0}(N) \rightarrow P(N, \tau)$, where

$$
P A(P-Q)=\left(\int_{Q}^{P} \phi_{1}, \ldots, \int_{Q}^{P} \phi_{g-1}\right) \quad\left(\bmod H_{1}^{-}(N, \mathbb{Z})\right) .
$$

Here $\left\{\phi_{1}, \ldots, \phi_{g-1}\right\}$ is a basis of $H_{-}^{0,1}(N)$ and $H_{1}^{-}(N, \mathbb{Z})$ is injected into $H_{-}^{1,0}(N)^{*}$ by integration. $P A$ is called the Abel-Prym's map.

Proposition 5. The following diagram is commutative

$$
\begin{array}{ccc}
L^{0} H_{1}^{-}(X) & \stackrel{u}{\longrightarrow} & J^{0} W_{-1} H_{1}^{-}(X) \\
\partial \downarrow \cong & & \nu \downarrow \cong \\
A & \stackrel{\mu}{\longrightarrow} & P(N, \tau)
\end{array}
$$

where $\nu$ is given by the identification of $W_{-1} H_{1}^{-}(X)$ with $H_{1}^{-}(N, \mathbb{Z})$ and by using the duality given by integration; $\mu$ is the Abel-Prym's map, restricted on $A \subset \operatorname{Div}^{0} N$.

Proof. Obviously $L^{0} H_{1}^{-}(X)=\mathrm{Gr}_{0}^{w} H_{1}^{-}(X)$ which is isomorphic to $A$ through $\partial$ (cf. Proposition 4).

$$
\begin{aligned}
J^{0} W_{-1} H_{1}^{-}(X) & =\frac{W_{-1} H_{1}^{-}(X) \otimes_{\mathbb{Z}} \mathbf{C}}{W_{-1} H_{1}^{-}(X)+F^{0} W_{-1} H_{1}^{-}(X)}=\frac{F^{1} H_{-}^{1}(X)^{*} \oplus \overline{F^{1} H_{-}^{1}(X)^{*}}}{W_{-1} H_{1}^{-}(X) \oplus \overline{F^{1} H_{-}^{1}(X)^{*}}} \\
& \cong \frac{F^{1} H_{-}^{1}(X)^{*}}{W_{-1} H_{1}^{-}(X)} \cong \frac{H_{-}^{1,0}(X)^{*}}{H_{1}^{-}(N, \mathbb{Z})} \cong P(N, \tau) .
\end{aligned}
$$

To calculate $\mu$ we introduce the well-known symplectic base of $H_{1}(N, \mathbb{Z})$ :

$$
\left\{a_{1}, b_{2} ; a_{g+1}, b_{g+1} ; \ldots ; a_{g-1}, b_{g-1} ; a_{2 g-1}, b_{2 g-1} ; a_{g}, b_{g}\right\}
$$

for which

$$
\begin{gathered}
\tau_{*}\left(a_{i}\right)=a_{i+g}, \quad \tau_{*}\left(b_{i}\right)=b_{i+g} \quad \text { for } i=1,2 \ldots, g-1 ; \\
\tau_{*}\left(a_{g}\right)=a_{g}, \quad \tau_{*}\left(b_{g}\right)=b_{g} .
\end{gathered}
$$

Furthermore, choose a basis $\left\{\omega^{1}, \ldots, \omega^{2 g-1}\right\}$ of $H^{1,0}(N)$ with $\int_{a_{i}} \omega^{j}=\delta_{i}^{j}$ for $i, j=1,2, \ldots, 2 g-1$.

Then $H_{-}^{1,0}(N)=\left(\omega^{1}-\omega^{g-1}\right) \cdot \mathbf{C} \oplus \cdots \oplus\left(\omega^{g-1}-\omega^{2 g-1}\right) \cdot \mathbf{C}$, hence

$$
H_{-}^{1,0}(N)^{*}=\left(\int_{a_{\mathrm{r}}-a_{g+1}}\right) \cdot \mathbf{C} \oplus \cdots \oplus\left(\int_{a_{g-1}-a_{2-1}}\right) \cdot \mathbf{C} .
$$

In this case $\mu$ is given as follows:

$$
\gamma \rightarrow\left[\sum_{i=1}^{g-1}\left(\int_{\Gamma}\left(\omega^{i}-\omega^{i+g}\right),\left(\int_{a_{i}-a_{i+g}}\right)\right],\right.
$$

which is exactly the map $\gamma \rightarrow P A(\gamma)$. Q.E.D. 
In fact using the same basis of $H^{1,0}(N)$ we have a map $j: P(N, \tau) \rightarrow J(N)$, where $J(N)$ is the Jacobi variety of $N$ and

$$
\begin{array}{r}
j \circ \mu(\gamma)= \\
{\left[\left(\int_{\Gamma}\left(\omega^{1}-\omega^{g+1}\right), \ldots, \int_{\Gamma}\left(\omega^{g-1}-\omega^{2 g-\mathrm{i}}\right), 0,-\int_{\Gamma}\left(\omega^{1}-\omega^{g+1}\right),\right.\right.} \\
\left.\ldots,-\int_{\Gamma}\left(\omega^{g-1}-\omega^{2 g-1}\right)\right] .
\end{array}
$$

It is clear that $j \circ \mu=(1-\tau) \circ A b$, where $A b: N \rightarrow J(N)$ is the Abel's map for $N$.

\section{Proof of TheOREM 8}

For the Abel's map $A b: N \rightarrow J(N)$ we have $(1-\tau) \circ A b(D)=A b((1-\tau) D)$ for each $D \in \operatorname{Div}^{0} N$. Since each generator of $A$ with minimal length has type $(1-\tau)(P-Q)$ then

$$
\begin{aligned}
j \circ \mu((1-\tau)(P-Q)) & =(1-\tau) \circ A b \circ(1-\tau)(P-Q)=A b \circ(1-\tau)^{2}(P-Q) \\
& =2 A b \circ(1-\tau)(P-Q)=2 P A(P-Q) .
\end{aligned}
$$

It follows that we must consider the map

$$
\Phi: N \times N \rightarrow P(N, \tau) \subset J(N), \quad(P, Q) \rightarrow 2 \cdot P A(P-Q) .
$$

Lemma 6. Let $N$ be a smooth projective curve with an involution $\tau$ without fixed points. If $N$ is neither a 4-, nor 8-sheeted covering of $\mathbf{P}^{1}$, then $\Phi\left(P_{1}, Q_{1}\right)=$ $\Phi\left(P_{2}, Q_{2}\right)$ if and only if: either $\left(P_{1}, Q_{1}\right)=\left(P_{2}, Q_{2}\right)$ or $\left(P_{1}, Q_{1}\right)=\left(\tau\left(Q_{2}\right)\right.$, $\left.\tau\left(P_{2}\right)\right)$ or $\left(P_{1}, P_{2}\right)=\left(Q_{1}, Q_{2}\right)$.

Proof. $\Phi\left(P_{1}, Q_{1}\right)=\Phi\left(P_{2}, Q_{2}\right)$ iff $2 P A\left(P_{1}+Q_{2}-Q_{1}-P_{2}\right)=0$ in $J(N)$. We consider $D=2(1-\tau)\left(P_{1}+Q_{2}-P_{2}-Q_{1}\right)$ as an element of $\operatorname{Div}^{0} N$. If $D \neq 0$ then $D=D_{+}-D_{-}, D_{-}^{ \pm}>0, \operatorname{deg} D_{+}=\operatorname{deg} D_{-}=4$ or 8 and $\operatorname{supp} D_{+} \cap \operatorname{supp} D_{-}=\varnothing$. Since $A b(D)=0$ in $J(N)$, then by Abel's theorem for $N$ we conclude that there exists a map $N \rightarrow \mathbf{P}^{1}$ of degree 4 or 8 which is impossible by hypothesis. Thus $D=0$, which is possible only in the cases listed in the lemma. Q.E.D.

Theorem 7. Let $X$ be a curve of 1 , for which $N / \tau$ is a generic curve of genus $g(N / \tau) \geq 15$. Then using the mixed Hodge structure of $H_{1}^{-}(X, \mathbb{Z})$, constructed in 4 , we can get $N, \tau: N \rightarrow N$ and $\Omega$.

Proof. The mixed Hodge structure gives us $J^{0} W_{-1} H_{1}^{-}(X) \cong P(N, \tau)$. Using the generic Torelli theorem (V. Kanev [5], Friedman-Smith [4]) we obtain $N$ and $\phi: N \rightarrow M$, which gives us $(N, \tau)$. Let $R$ be the finite subset of $L^{0} H_{1}^{-}(X)$ defined as follows. If there exist elements $b$ of $L^{0} H_{1}^{-}(X)$ with $(b, b)=4$, then $R=\{a \mid(a, a)=4\}$. Otherwise put $R=\{a \mid(a, a)=8\}$ (see Lemma 1 ). Consider the 1-motive: $u: L^{0} H_{1}^{-}(X) \rightarrow J^{0} W_{-1} H_{1}^{-}(X)$. Since $g(N / \tau) \geq 15$ then $N / \tau$ is not a covering of $\mathbf{P}^{1}$ of degree 4 or 8 (see [1, p. 214]) implying $N$ 
is not a covering of $\mathbf{P}^{1}$ of degree 4 or 8 . Then by Proposition 5 and Lemma 6 the set $u(R)$ uniquely determines the set $\Omega$. Q.E.D.

Theorem 8. Let $X$ satisfy the conditions of Theorem 7. Suppose $X$ has only one singular point. Then $X$ and $\sigma$ are uniquely determined by the PMHS defined in 4.

Proof. By using Theorem 7 we reconstruct $N$, $\tau$, and $\Omega$; since $X$ has an ordinary singular point, which is obtained by identification of the points of $\Omega$, we recover $X$ and $\sigma$. Q.E.D.

\section{ACKNOWLEDGMENTS}

It is my pleasure to express gratitude to $\mathrm{V}$. Kanev who posed the problem of this paper and whose suggestions contributed to the simplifying of the primary proof.

I would also like to thank the referee for his useful comments and suggestions which helped to clarify the exposition.

\section{REFERENCES}

1. E. Arbarello, M. Cornalba, P. Griffiths, and J. Harris, Geometry of algebraic curves, vol.1, Springer-Verlag, New York, 1985.

2. J. Carlson, Extensions of mixed Hodge structures, Jouruées de Géomátrie Algébriqué d'Angers 1979, Sijthhoff and Noordhoff, 1980, pp. 107-128.

3. C. Clemens, $A$ scrapbook of complex curve theory, Plenum Press, 1980.

4. R. Friedman and R. Smith, The generic Torelli theorem for the Prym map, Invent. Math. 67 (1982), 473-490.

5. V. Kanev, The global generic Torelli theorem for Prym varieties, Izvestia Acad. Nauk USSR, 46 (1982), 244-268.

Department of Mathematics and Informatics, Sofia University, A. IVanov St. 5, Sofia 1126 BULGaRIA

Current address: Department of Algebra, Steklov Mathematical Institute, Vavilova 42, Moscow GSP-1 117966 USSR 